\title{
VALIDATION OF A NUMERICAL MODEL FOR FLOW AND BEDFORM DYNAMICS
}

\author{
Sanjay GIRI ${ }^{1}$ and Yasuyuki SHIMIZU² \\ ${ }^{1}$ Student member of JSCE, JSPS Postdoc Research Fellow, Dept. of Hydraulic Research, Hokkaido University \\ (North 13, West 8, Kita-Ku, Sapporo 060, JAPAN) \\ ${ }^{2}$ Member of JSCE, Dr. Eng., Professor, Dept. of Hydraulic Research, Hokkaido University (North 13, West 8, \\ Kita-Ku, Sapporo 060, JAPAN)
}

\begin{abstract}
An explorative study has been carried out within the scope of this paper in order to validate a morphodynamic numerical model with the assessment of model sensitivity to some factors and parameters. The flow model component is a vertical two-dimensional with non-hydrostatic, unsteady free surface flow condition. The flow model has been coupled with sediment transport models. Two different sediment transport approaches have been used, namely Ashida \& Michiue's bedload transport formula and a stochastic pick up deposition formulation for non-equilibrium sediment transport proposed by Nakagawa \& Tsujimoto. The model performance has been tasted for different turbulence closures, namely a zero-equation, a standard $\mathrm{k}-\varepsilon$ and a non-linear $\mathrm{k}-\varepsilon$ models, in the context of morphodynamic simulation. Model performance has been evaluated for the prediction of temporal variation of flow-depth and boundary shear stress induced by bedform evolution based on experimental measurements.
\end{abstract}

Key Words: Morphodynamic model, turbulence model, river bedforms, sediment transport

\section{INTRODUCTION}

Flow and bedform dynamics in alluvial channels is one of the challenging problems in river engineering practice, despite many invaluable efforts that have been made so far. Prediction of morphodynamic feature of bedforms (ripples and dunes) as well as resistance to flow induced by them is of practical significance.

A comprehensive review of past investigations on river dunes has recently been done by Jim Best ${ }^{1)}$. $\mathrm{He}$ has pointed out towards the necessity for integrated and interdisciplinary studies in order to improve existing understanding on the kinematics of dune morphology and flow. Most past observations evinced strong correlation between turbulence structures induced by flow separation, bed resistance and sediment transport. Consequently, all these aspects are equally important for quantitative determination of bedform morphodynamics. Of most past investigations, significant attempts were made to analyze flow and turbulence structures over fixed bedforms ${ }^{2}$. Best et al. ${ }^{3)}$ carried out laboratory measurements to evaluate influence of sediment transport on turbulence production at low transport rate. Schmeeckle et al. ${ }^{4)}$ conducted laboratory visualization of turbulence and suspended sediment transport over two-dimensional dunes. Schindler and Robert ${ }^{5)}$ examined flow and turbulence characteristics across the ripple-dune transition in laboratory flume. These observations have corroborated the significance of fluid dynamics of bedforms. Variety of approaches was developed in order to analyze bedform-induced morphology in alluvial channels and development of dunes. All these studies revealed influence of temporally dynamic parameter contributing to bedform development. A study on instability of sand bed was conducted based on a concept of granular material dynamics $^{6)}$, in which formation of small ridges from a random field of perturbation was simulated using, so called, 'Movable Bed Simulator'.

Despite significant amount of past investigations, almost all of them are unable to tackle the complexity of temporal bed evolution and associated flow-field modification. Recently, we proposed a vertical two-dimensional flow model with a sediment pick up deposition function ${ }^{7), 8}$. It was shown that model possesses capability to simulate flow and turbulence field over fixed dunes. 
Model was able to replicate bedform evolution from an initial perturbation in physically based manner. Most field and laboratory observations on bedform development have revealed the dynamic pattern of bedform evolution with superimposition or amalgamation. We confirmed in our study ${ }^{8)}$ that the simulation of bedform evolution mechanism and their geometric characteristics can significantly be improved by using non-uniform pattern of vertical grid distribution. In present study, effect of turbulence closures and sediment transport models on simulation results has been evaluated. Three different turbulence closures and two different sediment transport models (equilibrium and non-equilibrium) have been used in order to assess their applicability for the simulation of bedform evolution process. Additionally, we demonstrate herein the model capability to replicate evolution of flow-depth and boundary shear stress induced by bedforms based on experimental measurements.

\section{BRIEF DESCRIPTION OF MORPHO- DYNAMIC MODEL}

A three-dimensional model with direct numerical simulation as proposed by Shimizu et al. ${ }^{9)}$ seems to be more complete approach for the computation of flow and turbulence over bedforms. However, implication of this flow solver with a sediment transport model for morphodynamic simulation may inhibit it from being applied efficiently because of extremely high computational effort. Consequently, aforementioned three-dimensional hydrodynamic model has been simplified to a vertical two-dimensional and enhanced by imposing non-hydrostatic, free surface flow condition and, subsequently, coupled with a couple of sediment transport modules.

Computation of time-dependent water surface change is of importance for realistic reproduction of free surface flow over migrating bedforms. Basically, majority of morphologic numerical models developed earlier assume rigid lid water surface condition to achieve numerically stable solution. Such model cannot reproduce properly flow characteristics and water depth variation induced by bedforms. In our study, we succeeded to achieve stable and reasonable solution under free surface flow condition over migrating bedforms. The kinematic condition, which constraints fluid particles to remain on the water surface at any time following the local flow velocity, is imposed along the water surface in order to compute water surface variation ${ }^{8)}$.

A no-slip boundary condition at the bed was employed; particularly a logarithmic expression for near-bed region was adopted. The periodic boundary condition was employed in this computation, that is, out put at the downstream end is set to be input at the upstream. The computation was performed for different length of calculation domain in order to assess the sensitivity of periodic boundary condition.

The equations were transformed into a boundary fitted coordinate system. Transformed equations were numerically solved by splitting them into non-advection and pure advection phase. Non-advection phase was computed using central difference method. The pressure term was resolved using SOR method. Advection phase was calculated using a high-order Godunov scheme known as CIP method $^{10)}$.

In our previous study ${ }^{8)}$, a nonlinear $\mathrm{k}-\varepsilon$ turbulence closure was employed that enables the anisotropy of Reynolds stresses to be considered to some extent. In a conventional $\mathrm{k}-\varepsilon$ model turbulence stress tensors are evaluated using linear relationship. In order to reproduce turbulence characteristics more precisely in shear flows with separation zone, a nonlinear term is added to the standard k- $\varepsilon$ model. Kimura \& Hosoda ${ }^{11)}$ made detailed analysis and comparison of a nonlinear $\mathrm{k}-\varepsilon$ model with other turbulence closures and found it more efficient than RSM or LES model in terms of CPU time. In present study, we have tested the performance of a standard k- $\varepsilon$ model and also a zero-equation model so as to assess the significance of turbulence closure in the context of morphodynamic simulation.

With regard to sediment transport model, two different approaches have been employed in this model. In our previous studies, we used a pick up deposition model for non-equlibrium sediment transport proposed by Nakagawa \& Tsujimoto ${ }^{12)}$. In present model, a module for equilibrium sediment transport proposed by Ahisda \& Michiue ${ }^{13)}$ has also been incorporated in order to assess its applicability.

\section{EXPERIMENT}

Venditti et al. ${ }^{14)}$ performed series of experiment to reproduce two-dimensional dunes. Along with the bedform evolution process, they measured variation of flow-depth and water surface slope at different time interval of bedform evolution. The rise in the water surface was measured using two water level sensors. They reported that initially water surface dropped several millimetres when flow was initiated. However, within a few minutes bedforms developed that increased resistance to flow, causing a rise in the water surface that varied amongst runs, but generally decreased with flow strength. An example of measurement record of water surface 

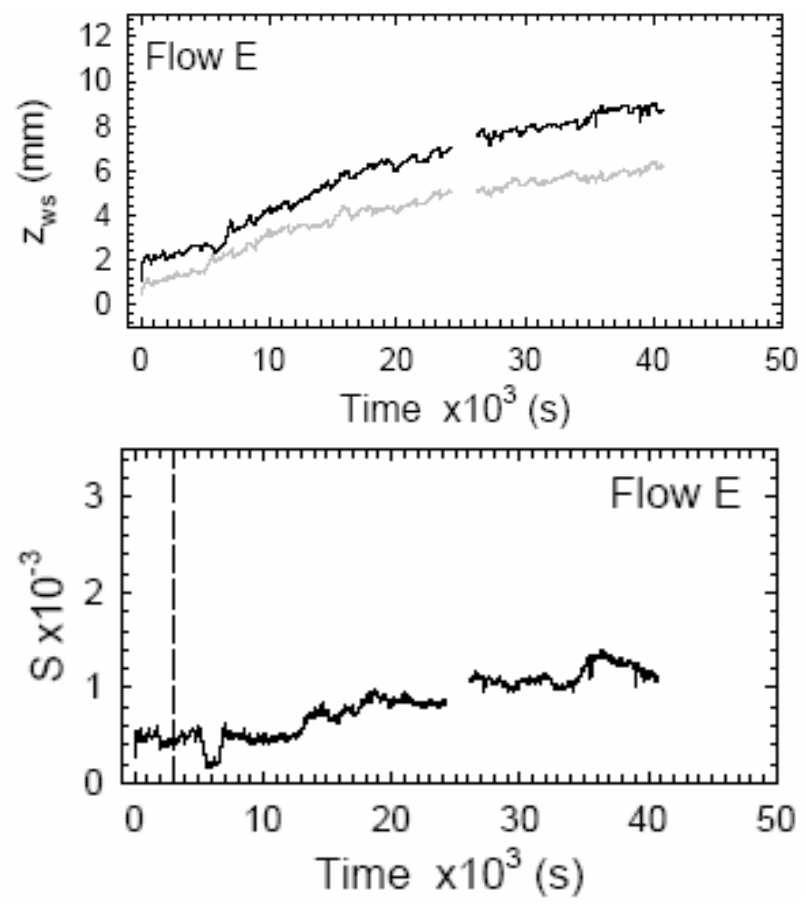

Fig.1 Measurement of flow-depth variation (top) and water surface slope fluctuation (bottom)

fluctuation $\left(Z_{w s}\right)$ can be seen in Fig.1 (top). With growing bedform size, water surface continued to increase. It is expected that water surface would increase asymptotically and reach an equilibrium value after some time.

In addition, they recorded temporal change of water surface slope. A typical example of water surface slope variation $(S)$ can be seen in Fig.1 (bottom).Using these data, one can easily determine the change of boundary shear stress as a function of time using depth-slope product.

All five cases and condition of their experiments can be found elsewhere ${ }^{8)}$.

\section{MODEL VALIDATION SENSITIVITY}

AND

\section{(1) Dune-induced flow-depth and boundary shear stress}

Flow resistance is associated with skin friction of sediment particles and form drag exerted by bed forms. In case of flat-bed, effective shear stress is equivalent to the grain shear stress. Contribution of form drag becomes significant in the presence of bedforms due to the flow separation and pressure variation. An adequate determination of bedform-induced resistance to flow that accompanies flow-depth variation is essential from practical engineering point of view. Proposed model is able to replicate bed shear stress variation associated with form drag exerted by temporal growth of bedforms. A quantitative comparison has
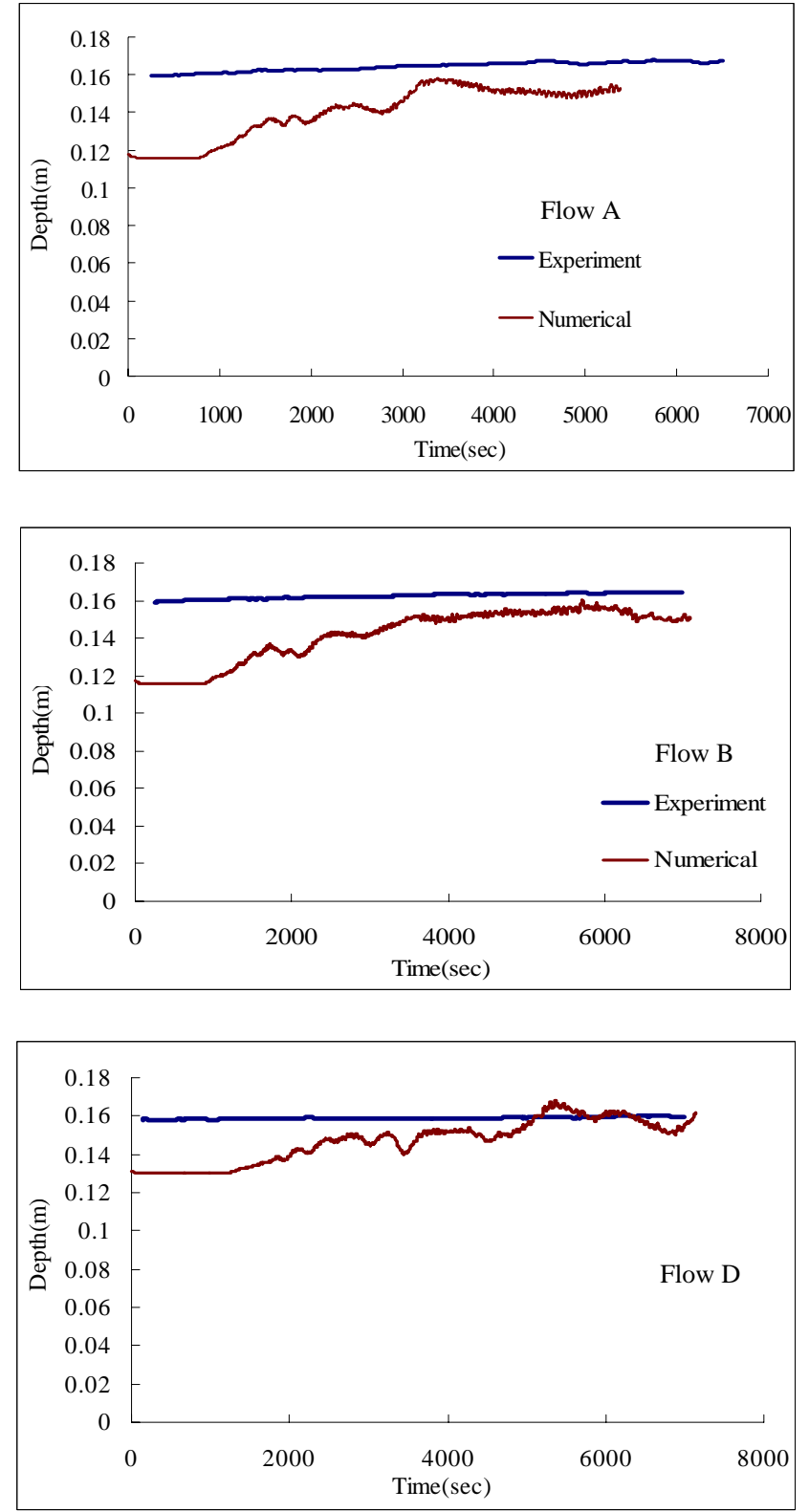

Fig.2 Comparison of flow-depth variation with respect to time

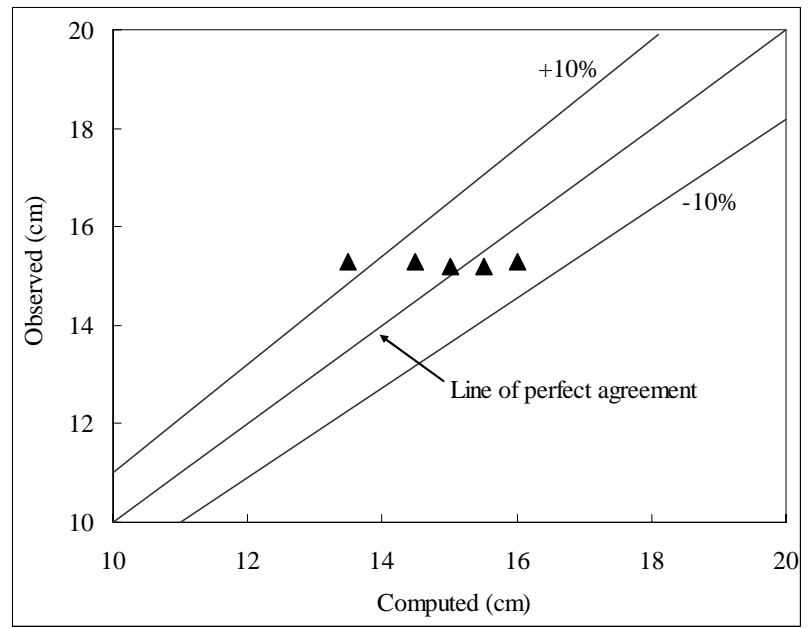

Fig.3 Comparison of equilibrium flow-depth 


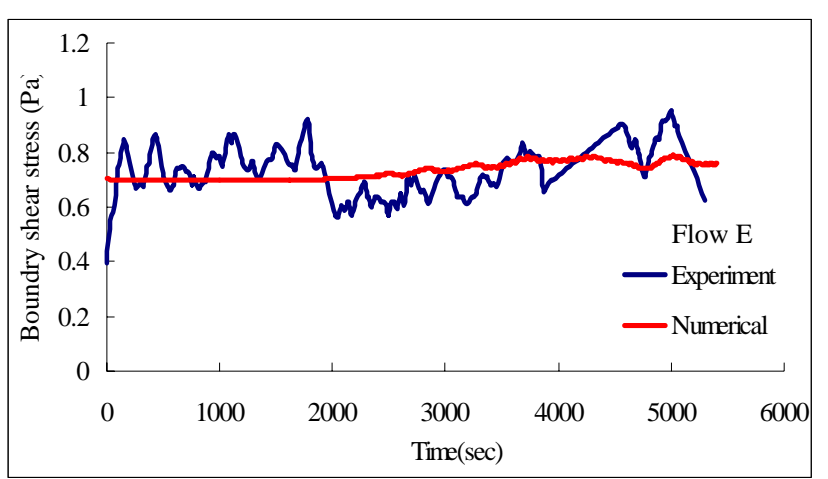

Fig.4 Comparison of boundary shear stress variation with respect to time.

been made to evaluate the model simulation on bed form-induced flow-depth variation (Fig.2). From the result, it can be seen that model underpredicted the flow-depth in the initial stage (particularly for the run with high flow intensity). It was found that the dune starts to form slowly in the beginning in numerical computation that may cause the underprediction of flow-depth in this stage. Also, the temporal variation of flow-depth in experiment appears to be rather low. In fact, it is rather difficult to measure precisely the change in flow-depth in laboratory experiments, particularly in the starting stage as it usually grows rapidly.

However, in latter stage, the flow-depth was found to be predicted with good accuracy (error is 5-10\%). A comparison with equilibrium flow-depth for all five experimental cases has been shown in Fig.3. Since, the shear stress is calculated from depth-slope product, it also shows the same tendency as depth variation. A typical comparison has been shown in Fig.4. It should be noted that a constant slope was used in numerical computation, whereas for the experimental case, the slope was measured as a function of time. Consequently, the boundary shear stress appears to be fluctuating in experiment.

A typical example of evolution of physical characteristics associated with bedform evolution can be seen in an instantaneous plotting depicted in Fig.6 (sub-section 3).

\section{(2) Evaluation of bedform simulation result} using different turbulence models

An attempt was made to assess the effect of turbulence models on bedform morphodynamic simulation. We used three types of turbulence closures in this computation, namely nonlinear $\mathrm{k}-\varepsilon$, standard $\mathrm{k}-\varepsilon$ and zero-equation models. It was found that there is no noticeable distinction between simulation results with standard and nonlinear $k-\varepsilon$ closures, in particular for morphodynamic simulation. From Fig.5, it can be seen that feature of
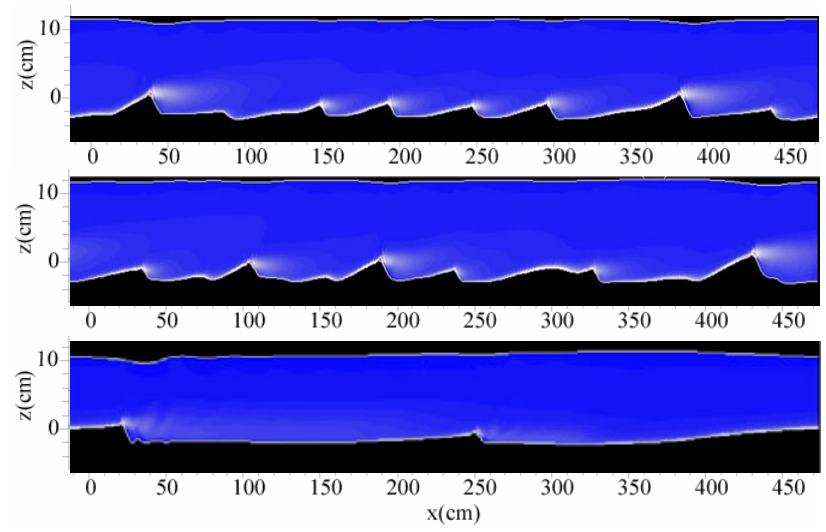

Fig.5 Simulation with a non-linear k- $\varepsilon$ (top), a standard $\mathrm{k}-\varepsilon$ (middle) and a zero-equation models (bottom).

bedforms differs very slightly and cannot be made any quantitative distinction. For the sake of comparison, some simulations were conducted with zero-equation turbulence closure under similar condition. From the simulation result (bottom plot of Fig.5), it can be inferred that model could not reproduce bedform evolution process adequately. Since a zero-equation model assumes local equilibrium of the turbulent and mean flow, it is usually inaccurate in separation region or in boundary layers subjected to extremely strong accelerations. Whereas, two-equation models take explicit account of the history of the turbulence through two transport equations and, thus offer good prediction of the characteristics of separated flows ${ }^{8)}$.

\section{(3) Influence of sediment transport model on} simulation of bedform characteristics

We attempted to evaluate the performance of our morphodynamic model using different sediment transport modules. Apart from a stochastic formulation of sediment pick up deposition model proposed by Nakagawa \& Tsujimoto, a bedload transport formula proposed by Ashida \& Michiue was also incorporated in the present model.

From an instantaneous feature of simulation result (Fig.6), it can be observed that model poorly predicts all characteristics associated with bedform evolution process when used Ashida-Michiue's sediment transport formula. The simulated bedform characteristics appear to be incompatible with physical observation. The flow-depth was also overestimated. This evinces inappropriateness of such approach that is based on equilibrium sediment transport. Whereas, simulation result with Nakagawa \& Tsujimoto's model showed realistic prediction of all physical characteristic ${ }^{8)}$. This leads to the conclusion that a non-equilibrium model, which can replicate phase lag between bed shear stress and particle transport due to imposition of step length, is an appropriate approach for simulating bedform morphodynamics. 

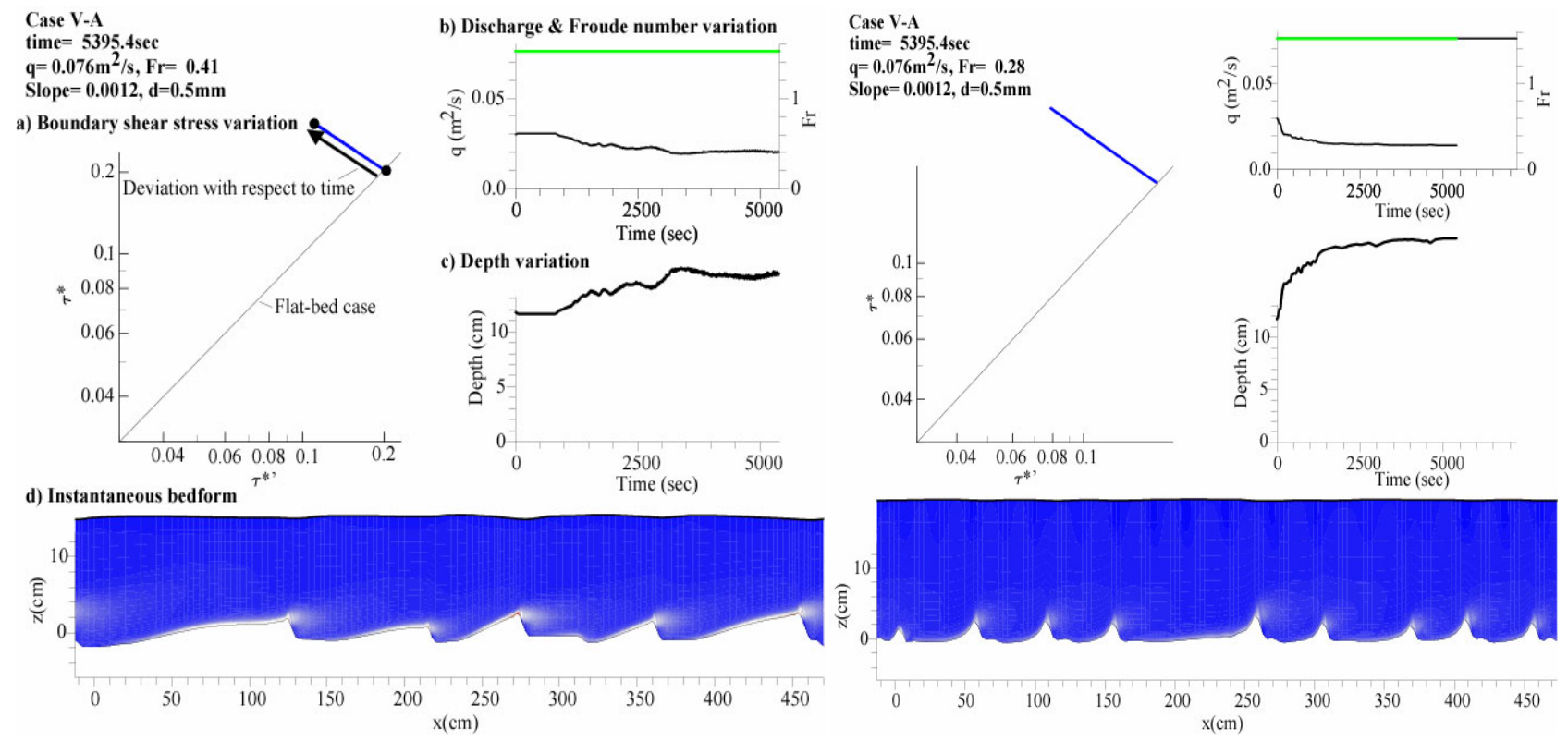

Fig.6 Instantaneous feature of numerical simulation result with Nakgawa \& Tsujimoto's (left) and Ashida \& Michiue’s (right) sediment transport approach ( $\tau^{*}=$ effective bed shear stress, $\tau^{*}$ '=grain shear stress, $q=$ unit discharge).
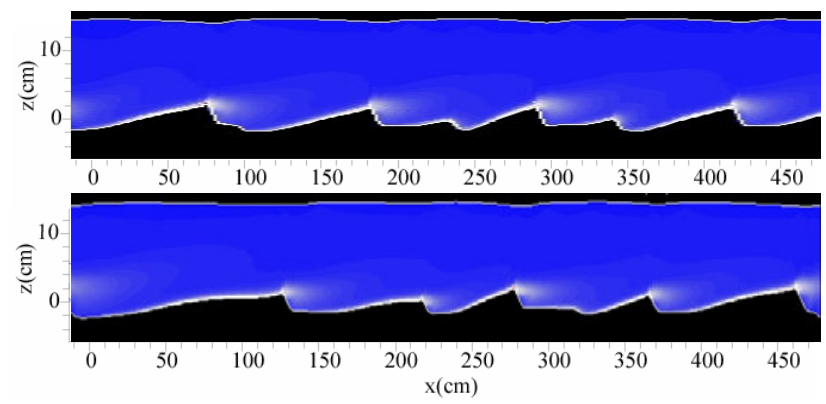

Fig.7 Instantaneous simulation result with $15.2 \mathrm{~m}$ (top) and $5 \mathrm{~m}$ domain length (bottom)
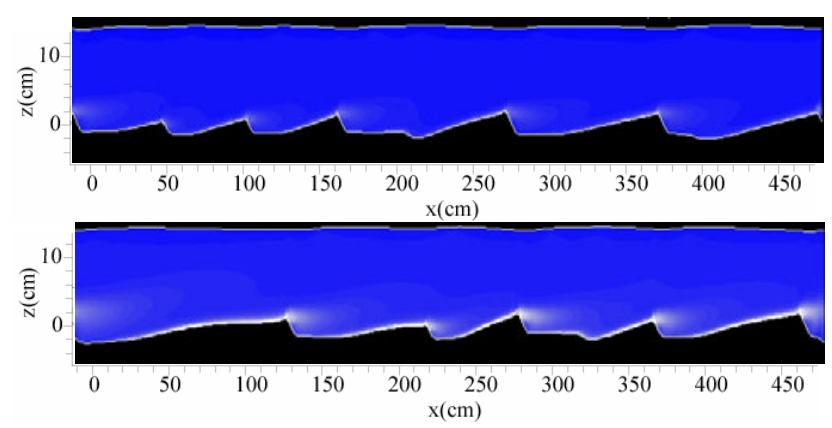

Fig.8 Simulation with random field of initial perturbation (top) and perturbation based on K-H instability (bottom)

\section{(4) Model sensitivity to domain length}

Since a periodic boundary condition has been employed in present computation, it is necessary to assess the effect of length of calculation domain in order to confirm the periodic nature of the phenomenon. We used two different domain length for one of the experimental cases (case A), namely $5 \mathrm{~m}$ and full length of flume (15.2m).The quantitative comparison of an instantaneous feature of bedforms has been illustrated in Fig.7. From this comparison, it can be inferred that model is almost insensitive to the size of calculation domain.

\section{(5) Model sensitivity to initial perturbation}

Initially, the computation of bedform evolution was performed by adding a very small random field of perturbation onto the sand bed. Venditti et al. ${ }^{15)}$ ascribed the initiation process to a Kelvin-Helmholtz instability and reintroduced a simple quantitative model for hydrodynamic instability to explain bedform initiation and validated with the results of their experiments. They proposed a relationship for interfacial instability to determine the initial wavelength at which the interface becomes unstable. We used the K-H model proposed by them to set the initial wavelength as an initial sinusoidal perturbation and then allowed it to grow using our simulation technique. We tested this idea for one of the experimental cases (run A). The result is depicted in Fig.8. Some small alteration can be noticed in size of bedforms; however no any significant quantitative distinction can be made between these two cases at this stage.

\section{(6) Model sensitivity to mean step-length}

In Nakagawa \& Tsujimoto's sediment transport model, the deposition rate is calculated based on the exponential distribution function of mean step-length. They proposed the value of mean steplength within the range of (50-250)d (where, $d=$ sediment diameter). However, we found that for higher value of mean step-length, the perturbation disappears and no bedform appears with the increment of time (particularly for the value more than 100d). It is well-known that Einstein proposed this value to be $100 \mathrm{~d}$. So, we used this value for all 
simulation. It should be noted that once sand waves start to form, this value has no effect on evolution of bedform characteristics (shape and size) and their equilibrium feature for this particular case. However, the influence of this sediment parameter on bedform initiation and evolution process as well as its physical description should be explored more thoroughly for the range of hydraulic conditions.

\section{CONCLUSIONS}

The performance of a vertical two-dimensional morphodynamic computational model was evaluated. Sensitivity of model performance to different factors and parameters was also assessed. From this study, the following conclusions can be drawn:

1) Model possesses ability to replicate temporal variation of physical characteristics associated with bedform evolution, such as temporal variation of boundary shear stress and flow-depth. Model underpredicts the depth variation in initial stage, particularly for the runs with higher flow intensity. However, model appears to be accurate enough to replicate equilibrium depth.

2) There is almost no difference between simulation results on bedform features using standard and non-linear $\mathrm{k}-\varepsilon$ turbulence models. However, a conventional zero-equation model, that simply assumes local equilibrium of the turbulent and mean flow, does not seem to be appropriate for such a case with abrupt change of mean property in separated flows as in case of simulating bedform dynamics. Moreover, this model severely underpredicts the turbulence characteristics (not shown in this study). The relevance of the turbulence models in the context of morphodynamic simulation should be explored more comprehensively in future studies.

3) Model performance significantly differs based on which sediment transport formulation is used. A stochastic pick up deposition model that accounts for non-equilibrium sediment transport seems to be more appropriate than conventional approach based on equilibrium sediment transport. However, influence of a sediment parameter (mean step-length) on bedform evolution process should be explored more thoroughly.

4) Model appears to be insensitive to the factors like domain length and initial perturbation.

Model results can be regarded as acceptable considering the complicated nature of the problem.

ACKNOWLEDGEMENT: This study is supported by JSPS Fellowship Program
(Grant-in-aid 17-05093). We gratefully acknowledge Jeremy Venditti for providing us supplementary experimental data.

\section{REFERENCES}

1) Best, J.L.: The fluid dynamics of river dunes: A review and some future research directions, J. Geoph. Res., Vol.110, doi:10.1029/2004JF000218, 2005.

2) Nelson, J.M., McLean, S.R. and Wolfe, S.R.: Mean flow and turbulence over two-dimensional bed forms, Water Resour. Res., Vol.29 (12), pp.3935-3953, 1993.

3) Best, J., Bennet, S., Bridge, J. and Leeder M..: Turbulence modulation and particle velocities over flat sand bed at low transport rate, J. Hydraul. Eng., ASCE, Vol.123 (12), pp.1118-1129, 1997.

4) Schmeeckle, M.W., Shimizu, Y., Hoshi, K. and Tateya, K.: Turbulent structures and suspended sediment over two-dimensional dunes, Proc. Int. Conf. Riv. Coast. Morph. Dyn., Genova, pp.261-270, 1999.

5) Schindler, R.J., and Robert, A.: Flow and turbulence structure over ripple-dune transition: an experiment under mobile bed condition, Sedimentology, Vol. 52, pp.627-649, 2005.

6) Gotoh, H., Harada, E. and Sakai, T.: Role of interparticle collision on instability of sand bed, Proc. $2^{\text {nd }}$ IAHR Symposium RCEM, pp.111-120, 2001.

7) Giri, S. and Shimizu, Y.: Computation of flow, turbulence and bed evolution with sand waves, Ann. J. Hydraul. Engrg, JSCE, Vol.50, pp. 169-174, 2006.

8) Giri, S., and Shimizu, Y.: Numerical computation of sand dune migration with free surface flow, Water Resour. Res., Vol.42, doi:10.1029/2005WR004588, 2006.

9) Shimizu, Y., Schmeeckle, M.W. and Nelson, J.M.: Direct numerical simulation of turbulence over two-dimensional dunes using CIP methods, J. Hydrosci. Hydr. Eng., Vol. 19 (2), pp.85-92, 2001.

10) Yabe, T., Ishikawa, T., Kadota, Y. and Ikeda, F.: A multidimensional cubic-interpolated pseudoparticle (CIP) method without time splitting technique for hyperbolic equations, PSJ, Vol.59, pp.2301-2304, 1990.

11) Kimura, I. and Hosoda, T.: A nonlinear k-e model with realizability for prediction of flows around bluff bodies, Int. J. Num. Meth. Fluids, Vol.42, pp.813-837, 2003.

12) Nakagawa, H. and Tsujimoto, T.: Sand bed instability due to bed-load motion, J. Hyd.Div., ASCE, 106, pp. 2029-2051.

13) Ashida, K. and Michiue, M.: Study on hydraulic resistance and bedload transport rate in alluvial streams, Transactions of JSCE, Vol. 206, pp. 59-69, 1972.

14) Venditti, J. G.: Initiation and development of sand dunes in river channels- A theses submitted in partial fulfillment of the requirements for the degree of doctor of philosophy, The University of British Columbia, pp. 1-291, 2003.

15) Venditti, J.G., Church, M., and Bennett, S.J.: On interfacial instability as a cause of transverse subcritical bedforms, Water Resour. Res., Vol. 42 (7), doi: 10.1029/2005WR004683, 2006. 